\title{
Application Of Devops For E-Commerce Website
}

\author{
Madhulika ${ }^{1}$, Namrata Dhanda ${ }^{2}$
}

Department of Computer Science and Engineering, Amity School of Engineering and Technology, Amity University Uttar Pradesh, Lucknow, India

Email: imadhulika18@gmail.com ${ }^{1}$, ndhanda@lko.amity.edu ${ }^{2}$

\begin{abstract}
In recent years, a big requirement for software developers has been the ability to deploy new application features fast, frequently and in short release cycle time. This requirement, however, is most relevant to an e-commerce. An e-commerce website refers to a web application that focuses on selling products or services over the internet, and as a part of the internet it has to be updated and improved in a manner which does not hinder its functioning and is achievable in an efficient and timely manner. The DevOps approach ensures this. DevOps (Development Operations) is a 3rd generation Software Development philosophy which creates a collaboration between IT Operations and Software Development. This results in a decrease in the amount of time required to develop and deploy any application as it employs the feature of continuous development. It uses the agile methodology as its basis. AGILE methodology is a practice that promotes continuous iteration of development and testing throughout the software development lifecycle of the project. Both development and testing activities are concurrent unlike the Waterfall model. Thus, DevOps is emerging as a new age technology crucial for a faster and more efficient website development. This paper focuses on the need of DevOps for the development of e-commerce website, its requirements and the benefits of using the DevOps in e-commerce website development.
\end{abstract}

Index Terms- Agile; continuous deployment; DevOps; software development; IT operations; Waterfall model

\section{INTRODUCTION}

In a world of rapid development and technological advancements, people have started relying heavily on the internet for many of their needs, including the need of buying things. From clothes to grocery, everything is available online now, and online shopping is slowly taking the art of trade to a whole new level. Any person with an access to the internet has taken part in some form of online shopping at a point. Online shopping has revolutionized in such a way that it is now a $\$ 1$ tn industry. A crucial element for the industry of online shopping is an e-commerce website. An ecommerce website is the way for a business to access the global market and spread its reach to the consumers of the world. Thus, in this day and age of rapid revolution of the online shopping world the need for efficient and robust e-commerce website has become more prominent than ever before. And what has become even more important is to overcome the challenges of the e-commerce platform as quickly as possible so as to keep the business functional without taking a lot of time to update or fix problems. DevOps is just the tool for that. DevOps is a modern day methodology which helps in developing websites and updating them in fast and easy manner. E-commerce website challenges that previously took up to several weeks or even months can be solved in a matter of hours or sometimes even minutes. DevOps follows Agile methodology at its core which promotes the feature of continuous development. DevOps is a very powerful tool that is redefining the limitations in the development of software and websites, and in an industry like the e-commerce business, where speed is the currency, DevOps offers solutions that would bring any business a step above the rest of the competition. Ecommerce giants like Amazon, Flipkart, Alibaba and Etsy have already embraced DevOps driven technical and business solutions for the development of their software.

\section{ECOMMERCE WEBSITE}

E-commerce or E-Business is the sale and purchase of goods and services over the internet. But as the name e-commerce or electronic commerce suggest the term applies to any electronic medium rather than just the internet, like the data or funds transferred electronically. Commonly it is known as online shopping. E-commerce websites are the medium through which e-commerce is conducted, i.e., an e-commerce website refers to a web application that focuses on selling products or services over the internet. Some examples of e-commerce websites are Amazon, Flipkart, Snapdeal, e-Bay, etc. Previously similar functions were performed through email or phone conversations, but with growing times, advancing technology and rapidly changing business landscape and with these development the need of a good web application with easy-to-use, efficient and robust features has grown exponentially. A good ecommerce website ensures user-friendly, clean and sharp design with fast loading time. But a major requirement for an e-commerce website to be considered good is the ability to be updated and improved in a manner which does not hinder its functioning and is achievable in an efficient and timely manner. In other words, as a part of the internet it has to be developed in a continuous fashion. [14]. 


\section{Available online at www.ijrat.org}

\section{DEVOPS}

The term DevOps was coined by Patrick Debois in a conference in 2009, which combines the terms software development (Dev) with IT operations (Ops) [1].

DevOps is a set of software development practices that when followed properly shortens the software development lifecycle (SDLC) while also delivering features, updates and fixes frequently. It works on the principles of agile methodology and features continuous development and deployment of software modules side by side. Instead of focusing on the one phase at a time like the waterfall model, it focuses on developing the software modules as a whole, deploying and testing after each phase and adding and updating features as and when required. It shares common goals with continuous delivery. [9]

DevOps shares a common background with lean thinking and agile methodology which will be discussed later.

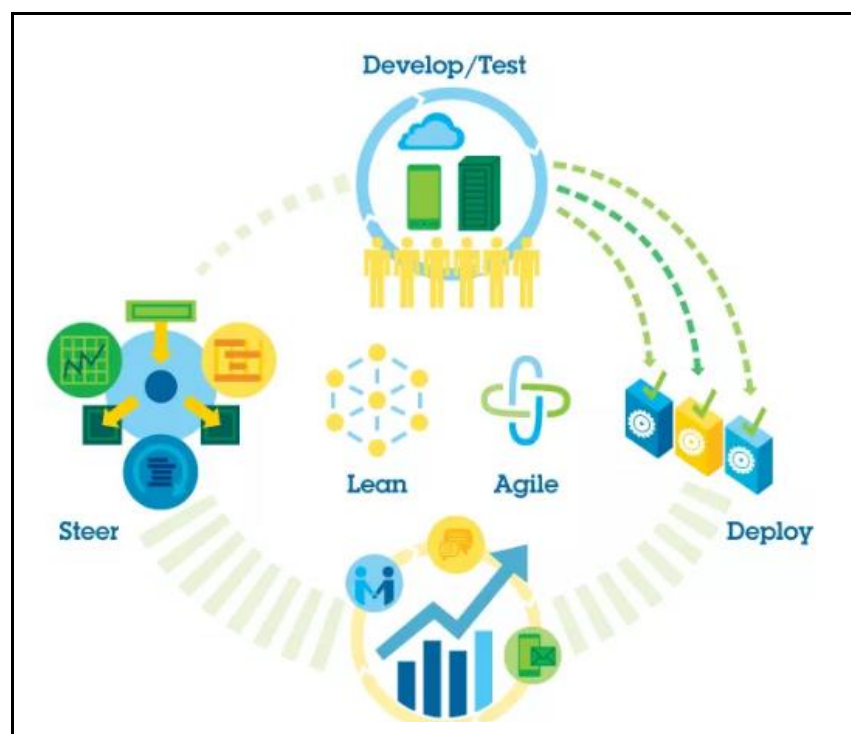

Fig. 1. Continuous process of DevOps [4]

\subsection{The need of DevOps in E-commerce}

As discussed before (refer section 2), a major requirement for an e-commerce website is the ability to be updated and improved in a manner which does not hinder its functioning and is achievable in an efficient and timely manner which is exactly what DevOps deliver, a way for fast and continuous development and deployment without going through each phase of software development for the whole web application. Not only does it produce faster fixes and updates, it also promotes better communication and collaboration of the development team resulting in higher-quality, outstanding and reliable product and services [10].

\section{AGILE METHODOLOGY}

AGILE software development methodology is a practice that promotes continuous iteration of development and testing throughout the software development lifecycle (SDLC) of the project. Both development and testing activities are concurrent unlike the Waterfall model. It is an iterative approach of development where instead of having different development phases, all phases are time-boxed into phases called sprints. These sprints usually span weeks at a time and each sprint develops the product as a whole instead of developing it in parts and after each sprints the software is deployed and tested. The sprints are focused around a list of deliverables which have to be achieved by the end of the sprint failing which work is reprioritized by taking information from the previous sprint. [5]

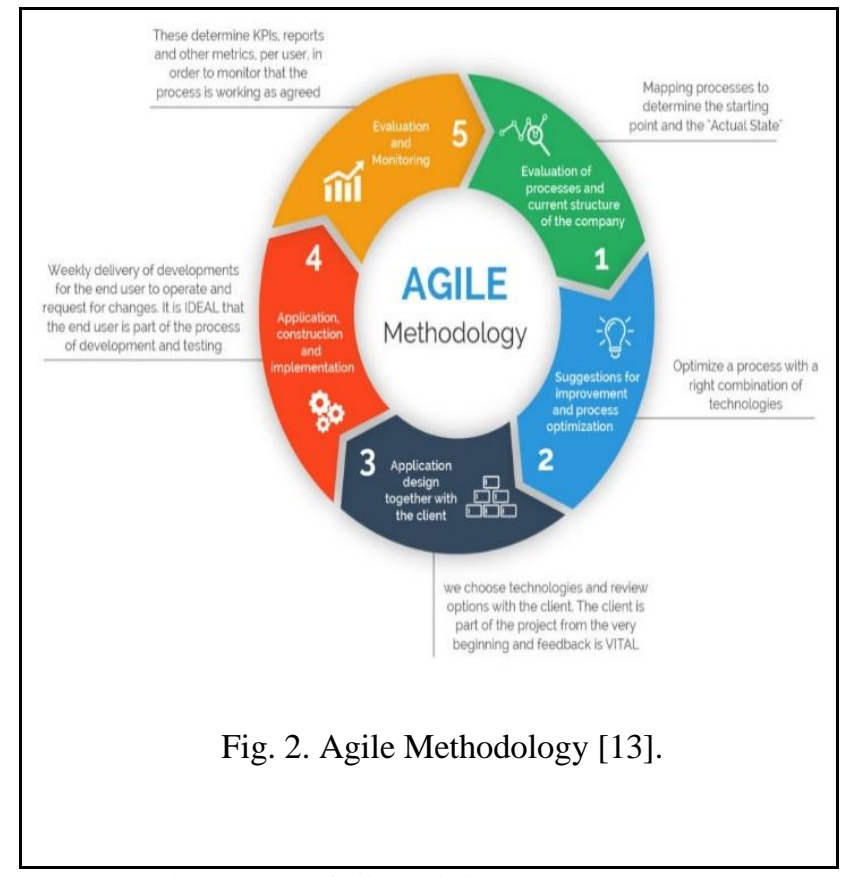

\subsection{Agile vs Waterfall model}

The difference in Agile and Waterfall methodologies is the approach to testing and quality of the software.

Waterfall model is a linear software development approach. It consists of a number of phases which have to be followed sequentially and each phase has be complete before the next phase can begin. No overlapping of phases occurs at any point and neither any backtracking can be done, i.e., going back to a certain phase after completing it to make improvement in it. The waterfall model is a very simple and relatively efficient Process model and it was the first SDLC approach. Due to its simplicity, straightforwardness and sure results it is still very famous amongst software developers and used as one of the main approaches of SDLC. Following are the phases of the waterfall model:

- Requirements: The initial requirements are gathered from the client; what is to be designed, 
International Journal of Research in Advent Technology, Vol.7, No.3, April 2019

$$
\text { E-ISSN: 2321-9637 }
$$

\section{Available online at www.ijrat.org}

its functions and purpose. Input and output requirements gathered.

- System Design: A system design is created with the help of the requirements gathered, helps in identifying hardware and system requirement. Code developed on pen and paper.

- Implementation: Code developed in system design phase is implement on the system.

- Integration and Testing: Units developed in the implementation phase are tested and integrated in the system.

- Deployment of System: Once the testing is successful the product is deployed in the market for the user

- Maintenance: After installation, any modifications or changes come under the maintenance phase.

Overall, the waterfall lifecycle model can be said to have two main phases, a design phase and a testing phase. The software is first built during design phase and only once it is complete fully does the testing phase begins. If any change, fix or update is required after the testing phase, the whole design phase is followed once again to implement the change. [5]

On the other hand, testing and programming are done in the same sprint or iteration. At each iteration a small piece of software is deployed and tested and its value is evaluated. By the end of all sprints the whole software is developed and is ready to be deployed in the market.
Table 1. Agile vs. Waterfall

\begin{tabular}{|c|c|}
\hline Agile & Waterfall \\
\hline $\begin{array}{c}\text { It is adaptive and iterative } \\
\text { change }\end{array}$ & It is predictive and sequential \\
\hline $\begin{array}{c}\text { Continuously responsive to } \\
\text { in a recursive and incremental } \\
\text { form }\end{array}$ & $\begin{array}{c}\text { Less responsive to change } \\
\text { at the end of the process, i.e., } \\
\text { one large release of software. }\end{array}$ \\
\hline $\begin{array}{c}\text { Requirements are gathered by } \\
\text { interaction through the } \\
\text { business users in the early } \\
\text { stages of the development } \\
\text { process during the } \\
\text { requirements phase and } \\
\text { cannot be changed easily in } \\
\text { later phases. }\end{array}$ & $\begin{array}{c}\text { A continuous communication } \\
\text { with the business users is } \\
\text { maintained throughout the } \\
\text { development process and } \\
\text { requirements are updated easily } \\
\text { when required through }\end{array}$ \\
$\begin{array}{c}\text { implementing them in the next } \\
\text { iterstion. }\end{array}$ \\
$\begin{array}{c}\text { Detailed documentation of } \\
\text { development Most of the } \\
\text { and paper before } \\
\text { implementation. }\end{array}$ & $\begin{array}{c}\text { Hands-on approach is followed } \\
\text { with minimal documentation } \\
\text { only when required. }\end{array}$ \\
$\begin{array}{c}\text { Detailed planning is required } \\
\text { beforehand }\end{array}$ & $\begin{array}{c}\text { Planning is done before each } \\
\text { iteration and work is } \\
\text { reprioritized and re-planned } \\
\text { after each iteration as per the } \\
\text { requirements. }\end{array}$ \\
\hline
\end{tabular}

\section{DEVOPS AND AGILE}

DevOps shares its principles with the Agile methodology and treats as one of its core elements, however there are certain problems that DevOps has to first overcome before utilizing the agile methodology. As discussed, Agile methodology utilizes iteration approach for the development of software with frequent and small deployments at consistent amounts of time. Further it promotes collaboration which does normally is not suitable for the IT Operations developers. Here, it becomes problematic as DevOps is a combination of both software development and IT operations. The frequency of the deployment puts immense pressure on the operations side, which leads to a big gap in collaboration between development and operations. A degree of lack of feedback in agile, and the sheer complexity of the system makes it hard to determine the redundant elements of the system. [8] DevOps however has devised several tools to overcome these problems. 


\section{Available online at $w w w . i j r a t . o r g$}

Table 2. Problems of Agile and Solutions offered by DevOps. [6]

\begin{tabular}{|c|c|}
\hline $\begin{array}{c}\text { Problems with Agile } \\
\text { features to the customers } \\
\text { sometimes becomes a } \\
\text { common occurrence. }\end{array}$ & $\begin{array}{c}\text { Solutions offered by DevOps } \\
\text { DevOps tools help in testing } \\
\text { and releasing new features as } \\
\text { and when they are released. }\end{array}$ \\
\hline $\begin{array}{c}\text { Compatibility of completed } \\
\text { software modules is } \\
\text { questionable. }\end{array}$ & $\begin{array}{c}\text { Open interfaces and test } \\
\text { automations overcome these } \\
\text { problems. }\end{array}$ \\
\hline $\begin{array}{c}\text { Quality of old functions is } \\
\text { broken due to addition of } \\
\text { new features. }\end{array}$ & $\begin{array}{c}\text { Quality of existing functions is } \\
\text { ensured sue to automation and } \\
\text { the reduction of repetitive } \\
\text { manual work. }\end{array}$ \\
\hline $\begin{array}{c}\text { Predictability of } \\
\text { development of the software } \\
\text { is less, and goals and } \\
\text { deadlines are sometimes } \\
\text { missed. }\end{array}$ & $\begin{array}{c}\text { Predictability and transparency } \\
\text { of the software development } \\
\text { process is increased due to the } \\
\text { tools and procedures of } \\
\text { DevOps. }\end{array}$ \\
\hline $\begin{array}{c}\text { No collaboration between } \\
\text { Development and IT } \\
\text { operations team. }\end{array}$ & $\begin{array}{c}\text { Development and IT } \\
\text { operations team collaborate } \\
\text { and share responsibilities in a } \\
\text { unified working environment } \\
\text { to achieve a common goal. }\end{array}$ \\
\hline
\end{tabular}

\section{AREAS OF DEVOPS}

The approach of DevOps begins from certain key areas. Four dimensions or areas of DevOps were proposed by Humble and Molesky [2] initially: Culture, Automation, Measurement and Sharing. However, many sources have defined Lean as another area. Collectively these area are called as the "CALMS" model [3]:

- Culture

- Automation

- Lean

- Measurement

- $\quad$ Sharing
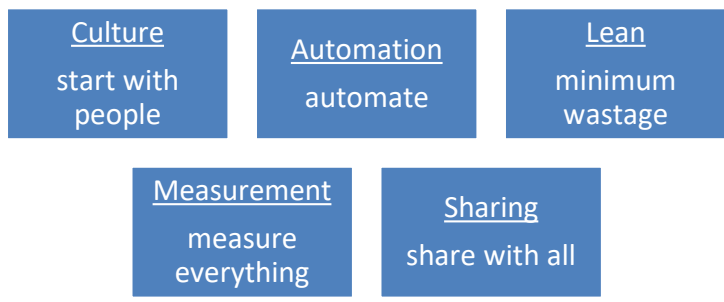

Sharing

share with all

Fig. 3. The CALMS model and its flow [3]

\subsection{Culture}

One of the biggest areas or aspects of DevOps is the organizational culture. The need for a strong collaboration between the software development team and the IT operations team is the main basis on which DevOps works upon. Thus, a good work culture in the organization is of the utmost importance where the development and operations teams can work together with a sense of equal responsibility and a unified goal [7].

\subsection{Automation}

Automation is the key of overcoming the problems that are faced by the Agile methodology. It helps in obtaining low lead times with quick feedbacks. It ensures a consistent and compatible development of the different software modules and also ensures the quality in case of addition of new features to an old function. [15]

\subsection{Lean}

Lean Thinking or Lean methodology is another building block of DevOps. It is a business methodology that aims towards finding solutions to challenges so as to maximize its benefits to the society and its value to individuals while minimizing or eliminating the waste produced in the process, i.e., development of products with as less of waste production as possible. In context of DevOps, Lean thinking is implemented by limiting Work-in-progress (WIP) and decreasing batch sizes. No extra work, which does not add to the value and quality of software is done while development. [6].

\subsection{Measurement}

Definition of measurement in DevOps given by Humble and Molesky [2] is "monitoring high-level business metrics such as revenue or end-to-end transactions per unit time". Measurement affects peoples way of work and thus it is crucial to visualize these metrics to give a clear view of how well the development process is going and how close to the end result is the software at a particular time. [2]

Application monitoring during production is also a part of measurement. In the traditional Waterfall model application monitoring is built downstream at the end of the development process. However in case of DevOps monitoring capabilities can be built in parallel which gives it a crucial advantage over the traditional Waterfall model.[11]

\subsection{Sharing}

A major component of collaboration between the development and operations team is Sharing. Sharing is a crucial component of the CALMS model as it promotes a transparent work environment and ensures better cooperation and understanding between the two teams. Sharing is also an excellent and efficient way of spreading knowledge and distributing responsibilities. This ensures the high-quality and reliability of the product. [6].

\section{ECOMMERCE AND DEVOPS}

As discussed, DevOps is an up and coming methodology which holds great benefits for ecommerce establishments:

\subsection{Culture}

E-commerce websites of these days have a lot of competition. This makes it extremely crucial for them to become progressive with the ability to deliver new features to the market at a rate that is faster than their 
International Journal of Research in Advent Technology, Vol.7, No.3, April 2019 E-ISSN: 2321-9637

\section{Available online at www.ijrat.org}

competitors. Organizations that fail to follow an iterative and continuous methodology for the development of their website are unable to perform transformational changes as traditional development lifecycles with prolonged period of development aim towards one large perfect website release thus losing the ability to improve and configure on a daily basis. The chances of a big perfect release is near to impossible, thus, making it an inefficient way of developing the website. [4]

As mentioned before, the traditional sequential SDLC approaches fall short on the progressive front as the product is neither deployed nor tested till the design phase is completed, and till then no feedback from the user can be gained. Not only does it hinder in the proper development of the software but also renders it incapable of introducing new changes, implementations, fixes or updates until the previous changes have been implemented. Thus, making it incapable of keeping up with the rapidly evolving market trends. This is a major disadvantage that DevOps overcomes [11].

Through ongoing improvement and its approaches of lean and agile approaches, DevOps has become the optimum solution for keeping up with the demands of progressive ecommerce businesses and facilitating continuous changes as and when required.[4].

\subsection{Automation}

As mentioned previously, Automation is crucial for the success of DevOps. Automation is the key of overcoming the problems that are faced by the Agile methodology. It helps in obtaining low lead times with quick feedbacks. It ensures a consistent and compatible development of the different software modules and also ensures the quality in case of addition of new features to an old function [6]. DevOps automates the processes of continuous integration, testing, deployment and release by dividing software module into small independent parts that are capable of being automated and when brought together fit compatibly with each other to create the complete software. This is a feature that was unheard in previous traditional SDLC.

Even though automation is critical to DevOps approach, it should be implemented carefully and only where required. Although automation makes a lot of tasks easier, if overused or implemented on elements which don't require it, it will end up making the system complex and hard to configure [4].

Automation provides tools and procedures that reduces workload, reduces costs and improves service reliability. It also provides frameworks for the businesses such that a systematic approach can be followed to replace and reduce waste processes with Faster DevOps enabled release cycles. Thus, implementing Lean methodology..

\subsection{Lean}

DevOps does not have a set rule of implementation, which means each organization can implement DevOps as they like. This means that as many variations of DevOps might be present as there are organizations that adopt them. [4]

Previously the software development and IT operations worked as individual teams without much cooperation or collaboration, but with DevOps the teams come together to serve a unified goal which helps in making the process of development faster and more reliable than ever. The cultural shift of working alone as individual teams to working together and Sharing resources and responsibilities is a major element of DevOps which helps in producing a rich and highly functional online end-user experience [12].

\section{CONCLUSION}

In conclusion, DevOps is the tool for that could overcome the ecommerce challenges that businesses are facing these days. The dynamic online ecommerce market demands a continuous state of evolution which results in the need for a tool like DevOps that could put out continuous deliveries and would provide the capability to the ecommerce business to innovate and improve with agility and speed to meet the customer demands and implement new facilities and features at a rapid pace.

The traditional waterfall model or other sequential SDLC cannot compare to the powerful and rapid development tools that DevOps provide which lets a business make modifications to an ecommerce web application in a matter of minutes to hours rather than weeks or months of the traditional lifecycles

The Lean and Agile approaches of DevOps not only helps with the continuous development, integration and delivery of web applications, but also lets the business produce reliable and efficient results with the least amount of wastage of resources.

Its key areas of cultural shift, Lean thinking, automation, measurement and sharing help in bringing it a step above the other software development processes. It can be used for a variety of other applications like security, testing, automation etc.

And though it is still a growing and evolving technology, DevOps is already making groundbreaking changes in the software development landscape, especially the ecommerce industries. Thus making it one of the most powerful technologies in the software development dynamics today and for the future.

\section{ACKNOWLEDGMENTS}

I am extremely grateful to my faculty guide, Prof. (Dr.) Namrata Dhanda (Professor, Department of Computer Science \& Engineering, ASET, Amity University Uttar Pradesh, Lucknow) for her valuable guidance, sincere co-operation, keen interest, encouragement and constructive suggestions which helped me in completing this review paper.

I am grateful to my guides and mentors at Amity University Uttar Pradesh, Lucknow, for their support and guidance 
International Journal of Research in Advent Technology, Vol.7, No.3, April 2019 E-ISSN: 2321-9637

Available online at www.ijrat.org

I am also grateful to any and all whose help, support and guidance have directly or indirectly resulted in the success of this review paper..

\section{REFERENCES}

[1] Debois, P. (2011). Devops: A software revolution in the making. Journal of Information Technology Management, 24(8), 3-39.

[2] Humble, J., \& Molesky, J. (2011). Why enterprises must adopt devops to enable continuous delivery. Cutter IT Journal, 24(8), 6.

[3] Riley, C. (2014) How to Keep CALMS and Release More! Logentries blog. Online. Available at: https://blog.logentries.com/2014/10/how-tokeep-calms-and-release-more/ [4] Editor. (2018) Three ways Devops can transform ecommerce business. Online. Available at: https://homebusinessmag.com/businesses/ecomme rce/site-management/three-ways-devops-cantransform-ecommerce-business/ [02.05.2018].

[5] Lotz, M. (2013). Waterfall vs. Agile: Which is the right development methodology for your project. Retrived online: http://www. seguetech. $\mathrm{com} /$ waterfall-vs-agile-methodology.

[6] Hamunen, J., (2016). Challenges in Adopting a Devops Approach to Software Development and Operations. Alto University school of Business, (2016).

[7] Earnshaw, A (2013) What is a Devops Engineer. Online. Available at https://puppet.com/blog/whata-devops-engineer.

[8] Menzel, G (2015) DevOps - The Future of Application Lifecycle Automation. A Capgemini Architecture Whitepaper - 2nd Edition

[9] Marie, T. (2014) What is DevOps? Even the Thought Leaders Can't Agree! Devops Zone. Online. Available

at: https://dzone.com/articles/what-devops-eventhought.

[10] Stewart, N (2016). How Devops Is Resetting The Ecommerce Landscape. Salmon, (2016)

[11] Wilsenach, R. (2015) DevOpsCulture. Online. Available http://martinfowler.com/bliki/DevOpsCulture.html

[12] Spikelab. The word Devops and a marketing problem. Online. Available at: http://www.spikelab.org/blog/the-word-devopsand-a-marketing-problem.html.

[13] Admin. (2018) Agile Project Management Methodology - Manifesto, Frameworks and Process. Online. Available at: https://backlinkme.net/project-management/agileproject-management-methodology/.

[14] Vangikar, P. (2018) What is e-Commerce? What are e-Commerce Websites?. Online. Available at: https://cyberchimps.com/e-commerce-websites/ .

[15] Nocera, D. I. F., Di Noia, T., \& Gallitelli, D. Innovative Techniques For Agile Development: Devops Methodology To Improve Software Production And Delivery Cycle. 